\title{
Mechanical, Morphological and Water Absorption Properties of Polypropylene Based Composites
}

${ }^{*}$ M.P.Jenarthanan, ${ }^{1}$ N G Ramkhi, ${ }^{2}$ M. Ramachandran, ${ }^{2}$ Vimala Saravanan

SASTRA Deemed University Thanjavur, India

REST Labs, Kaveripattinam, Krishnagiri, Tamil Nadu, India.

*Corresponding author Email: jenarthanan@mech.sastra.edu

\begin{abstract}
Polyethylene compounds are used instead of PE in many applications to improve the mechanical properties of PE (such as tensile strength, tensile modulus and tensile strength). Different types of combinations can be used to enhance these properties. Fibers are considered to be effective reinforcing fillers for PE. Plastic compound? Not all plastics are compounds. In fact, most plastics used in toys, water bottles and other familiar items are not composite. They are clean plastics. Polypropylene is not as solid as polyethylene. Polypropylene is harder than polyethylene and is resistant to chemicals and organic solvents. Polypropylene is pure, non-stretchable and much harder than polyethylene, commonly called fiberglass, in fact such a compound Also known As for glass-fiber reinforced plastic (GFRP), this is Specific to material properties such as tensile strength Made in different formats for permutations, stiffness, compressive strength and thermal expansion and different price points. For one, plastic deck boards are completely synthetic. It is considered safe in all plastics; it is a strong heat-resistant plastic. Due to its high heat tolerance, it is unlikely to leak even when exposed to hot or cold water. It is allowed to use with storage of food and beverages. Polypropylene (PP) is the most common in the world Is one of the thermoplastics used. Polypropylene plastic packaging, machinery and Plastic parts and threads for equipment and also used for textiles. Some plastic, BPA or other harmful chemicals in our body Or it could badly affect the world we live in. Polypropylene, a complex plastic, is common Considered safe for humans and these common ingredients are often the same Are used for products, but not theirs What are the differences? Polyester and polypropylene The main difference between the two is polyester Polypropylene is more water resistant than, It works well for athletic wear Is a quick-drying cloth. Plastic is a polymer with a large molecular weight. Polypropylene is an example of a plastic polymer. The main difference between polypropylene and plastic is that we can make crystal clear material out of polypropylene, while plastic material is generally not clear.
\end{abstract}

Keywords: Polypropylene, Mechanical properties, Morphological properties, Water absorption.

\section{Introduction}

Polypropylene and nylon (or polyamide) is two identical synthetic plastics with some important differences. Nylon is low friction, highly compatible and can withstand high temperatures, making it ideal for producing prototype and resistive components. Inhalation of microscopic particles can cause respiratory irritation. Smoke generated during thermal processing can cause reactions such as irritation, pneumonia and asthma. Environmental Risks: Polypropylene is considered biologically dull solid and non-toxic. Made of PVC synthetic Ingredients. But a mixture of wood and plastic Instead, PVC decking is $100 \%$ plastic. Like the mix, it reduces maintenance Common associated with wood mold and pests Designed to prevent problems infections. Polypro is one of the most widely produced types of plastic, which is generally cheaper overall. What is polypropylene made of? Polypropylene, also known as In the production of olefin, oil and natural gas Is a synthetic thermoplastic polymer derived from Until the late 1950s, gas was a by-product of propylene oil and natural gas facilities. Is Polypropylene Safe? Yes! Polypropylene rugs are completely safe because they do not contain harmful chemicals or substances. It is made of pure and soft plastic; Flexibility and softness are key features of polypropylene carpet and rugs. A recent study found that baby bottles made of polypropylene plastic emit millions of micro plastic particles. What children eat. Making the formula in a non-plastic container is one way to reduce your baby's exposure to micro plastics such as PVC and polystyrene, while converting plastic into baby bottles when cooled. Due to its relatively low emissions, it is often used for the manufacture of medical equipment and storage in museum collections; in addition, Polypropylene has excellent mechanical properties and fatigue, High resistance to impact, heat and frost Contains. It is very prone to corrosion and chemical leaks Resistant, solvents, bases and ideal for storing acids. Polypropylene is second only to polyethylene The most commonly used is plastic.

\section{Polypropylene}

PP92-96 can be prepared by polymerizing a thermoplastic polymer and Propylene molecules. It's in three main sources Retrieved from. Worldwide, most propylene Monomers from the vapor-cracking process using naphtha, a valuable component of crude oil Are coming. In general, the target product of the naphtha cracker is the ethylene monomer. Propylene is cracking Depending on a by-product of the process and the raw material Produced in different proportions. Oil raw material. Many cracking processes are closely linked to a propylene plant Naphtha effectively collects propylene from 
cracks.FRPCs are fibers and PP Are made by combining. A binder or 'matrix' and Keeps the fibers in place.PP has many useful properties such as high thermal decomposition temperature. Its expandable application Transparency, flame resistance, dimensional stability and high IS. Matrix subject, PP is widely used because it is a somewhat specialized natural fiber reinforced polypropylene compound that strengthens strength Natural through various treatments of natural fibers Better adhesion can be obtained between the fibers and the PP. Lignocelluloses is used as a reinforcing filler in thermoplastic polymer compounds and as a polypropylene matrix to determine its ability to be used as a rice-husk flour reinforcing compound to form particle-reinforced compounds. For thermoplastic polymer, four stages of filler loading are designed in the sample preparation to determine the experimental The body of the mixture according to the filler Data for mechanical and morphological properties load. Samples for the electron microscope were made from a carbon nanotube / polypropylene compound and mounted on an electron microscopy holder holding a standard scanning carbon tape. A thin layer of chromium was placed on the sample. Electron microscopic imaging of the nano compound under high vacuum was performed by a $10 \mathrm{kV}$ Philips XL30 ESEM instrument. The matrix polymer polypropylene (PP) used to make the composites has a density of $0.91 \mathrm{~g} / \mathrm{cm} 3$ and a melting flow index of $2 \mathrm{~g}$ at $8 \mathrm{~g} / 10 \mathrm{~min}$ and $2.16 \mathrm{~N}$ under load. Kinetic properties of the PP used: tensile strength 28.5 MPa, tensile modulus $1250 \mathrm{MPa}$, flexibility $38.5 \mathrm{MPa}$ and flexible modulus $1150 \mathrm{MPa}$ derived from Maleated polypropylene (MAPP) Eastman chemical, KO, mg. Melting point $158 \mathrm{C}$ and average molecular weight 52,000 .

\section{Mechanical properties}

Lignocelluloses material-thermoplastic polymer Mechanical properties and morphology of the compound Effect of compatible agents on. A particle-reinforced mixed rice-husk dough reinforcement filler and polypropylene is prepared using a thermoplastic matrix polymer and its mechanical and morphological properties are measured by the amount of compatibility agent used in the process. Its advantage is proper recycling of walnuts. However, Hydrophilic properties of lignocelluloses materials Due to their mechanical properties of these compounds slightly smaller than synthetic properties. Poor interface bonding between lignocelluloses Material and hydrophobic matrix polymer reduces the mechanical properties of the compounds. Let go of this problem using compatible agents. Become these compatible agents, one side is chemically bonded with the hydrophilic lignocelluloses filler, while the hydrophobic polymer helps to moisturize the chain, while Tensile strength and high brittleness The mechanical properties are greatly enhanced by the combination of Are improved, but also the compatibility Agent. Between the filler and the matrix Bad interface in Reduced tensile strength of the polymer composites, But tensile strength And modulus further updated because SFRP compounds are more attractive, simpler to imagine, more economical and have better Mechanical properties. SFRP compounds mostly regular though technologies, the relatively simple and inexpensive process for processing polymers involves the production of short fiber composites such as exhaust compound and needle formulation, compression design and more. Thermal analysis methods for fibers and polymers and analysis to classify the relationships between can provide an effective approach to doing. Longer CFs than shorter CFs in SCF / PP compounds Exhibiting excellent thermo-mechanical properties this study suggests. Increasing the length of carbon fiber Increases, the thermal stability of SCF / PP compounds Overall, the polymerization rate of the cellulose and the microfiber angle of thermal decomposition of the SCF / PP compounds improved the mechanical properties of the cellulose content in the fiber compared to the unfilled PP. Polymerization and low volume fibers exhibit high tensile strength and modulus of microfiber angle. The length of a single fiber expressing variations of these fibers in the machine is between the properties and the fibers. All natural fibers are hydrophilic cellulose molecules because they are strong hydroxyl groups.

\section{Morphological properties}

This study is on paddy-husk / polypropylene composites Physical, mechanical and to study morphological properties aims to explore everything. Various Kind of lignocelluloses based The possibilities for using the product are specific Applications that require desired physical and mechanical properties. Rice-husk / polypropylene composites, fiber loading, fiber size and binding agents and reinforcement Different mechanical, with different combinations of conditions It was planned by examining physical and morphological characteristics. The physical, mechanical and morphological properties of various rice-husk flour / polypropylene composites are studied using filler loading and binding agents. Four stages of filler loading are designed. To facilitate the contact between the fiber and the polypropylene matrix, structural binding agents were added to the compounds. Untreated compounds in the presence of large CNC agglomerates Studied the high and low effect of molecular weight PEOs on PLA / PEO / CNC compounds and different PEO / CNC ratios on crystalline, chemical, mechanical and morphological properties. Low molecular weight PEO has been found to be the most efficient nano scattering particles, but, on the contrary, plasticizes these PEO compounds and first dilutes their behavior into brittleness. No significant improvement in traction modulus and strength was reported. At the interface bond between the hydrophilic filler and the hydrophobic matrix polymer. The mechanical and morphological properties of the compound were explored at various test stages during loading and changing the size of the compliance agent used. A particle-reinforced compound was prepared using rice-husk dough reinforcement filler and polypropylene as a thermoplastic matrix polymer and its mechanical and morphological properties were measured by the active adhesive agent.

\section{Water Absorption}


Hydride polypropylene (PP) compounds are produced by the injection process, which has their effect on the water absorption properties and the tensile properties of hemp / hemp fibers. The effect of the hybrid on water absorption and kinetics was estimated by immersing the hybrid mixture samples of hemp fiber in distilled water at 40, 60 different temperatures and absorbing $80 \mathrm{C}$ of moisture. Compounds showed physiological pattern distribution; However, an exception was found at higher temperatures and water absorption studies following the ASTM D570-98 method were carried out due to the dissolution of low molecular weight materials from the micro cracks and natural fibers formed at the interface. Six needle-shaped attack models immerse in distilled water at temperatures of 40, 60 and $80 \mathrm{C}$, respectively, and study the dynamics of water absorption. To study the effect of water absorption on mechanical properties, six traction test samples of each sample were immersed in distilled water at room temperature and periodically determined with higher or lower water content, as previously described. The water absorption curves (A-D) of the infused PP, hemp / fiber composites are shown at $40 \mathrm{C}$, whereas the square root of the soaking time is projected against the percentage of water absorbed. Each data point represents an average of six samples. In all samples, except PP, the percentage moisture absorption, Mt, increases steadily and is equal to the saturation point after $1 / 2$ of the initial phase $\mathrm{D}$, indicating a Ficcian diffusion pattern. Moisture absorption in compounds is recommended by three main features and they include. (i) the distribution of water molecules within the micro gaps between the polymer chains; (ii) Incomplete moisture and (iii) transport of water molecules through micro-cracks in the matrix and (iii) capillary transport of water molecules to gaps and imperfections in the interface between fibers and polymers. Although all three methods are active, the overall effect can be conveniently designed considering the diffusion mechanism. There are three different types of diffusion behaviors, of which I or physical localization, case II and three nonphysical or irregular localization phenomena may theoretically differ by the shape of the curve, represented by the empirical equation:

\section{Conclusion}

As a matrix material, PP is widely used because it is a somewhat specialized natural fiber reinforced polypropylene compound that strengthens strength through various treatments of natural fibers to obtain better adhesion between natural fibers and PP. Poor interface bonding between filler and matrix is caused by polymer compounds that reduce tensile strength, but tensile strength and modulus SFRP compounds are very attractive due to the simplicity, economy and excellent mechanical properties of the fiction. It was planned by examining the various mechanical, physical and morphological properties of different combinations of rice-husk / polypropylene composites, fiber loading, fiber size, and bonding agent and reinforcement conditions. However, an exception was found at higher temperatures and may have been due to micro cracks and water absorption studies generated at the interface following the dissolution of low molecular weight materials from ASTM D570-98 natural fibers. Method.

\section{Reference}

[1]. Shubhra, Quazi TH, A. K. M. M. Alam, and M. A. Quaiyyum. "Mechanical properties of polypropylene composites: A review." Journal of thermoplastic composite materials 26, no. 3 (2013): 362-391.

[2]. Chen, Xiaoya, QipengGuo, and Yongli Mi. "Bamboo fiber-reinforced polypropylene composites: A study of the mechanical properties." Journal of applied polymer science 69, no. 10 (1998): 1891-1899.

[3]. Yang, Han-Seung, Hyun-Joong Kim, Jungil Son, Hee-Jun Park, Bum-Jae Lee, and Taek-Sung Hwang. "Rice-husk flour filled polypropylene composites; mechanical and morphological study." Composite structures 63, no. 3-4 (2004): 305-312.

[4]. Assouline, E., A. Lustiger, A. H. Barber, C. A. Cooper, Eugenia Klein, Ellen Wachtel, and H. D. Wagner. "Nucleation ability of multiwall carbon nanotubes in polypropylene composites." Journal of Polymer Science Part B: Polymer Physics 41, no. 5 (2003): 520-527.

[5]. Ashori, Alireza, and Amir Nourbakhsh. "Reinforced polypropylene composites: effects of chemical compositions and particle size." Bioresource technology 101, no. 7 (2010): 2515-2519.

[6]. Joseph, P. V., K. Joseph, Sabu Thomas, C. K. S. Pillai, V. S. Prasad, GabriëlGroeninckx, and Mariana Sarkissova. "The thermal and crystallisation studies of short sisal fibre reinforced polypropylene composites." Composites Part A: Applied Science and Manufacturing 34, no. 3 (2003): 253-266.

[7]. Yang, Han-Seung, Hyun-Joong Kim, Hee-Jun Park, Bum-Jae Lee, and Taek-Sung Hwang. "Effect of compatibilizing agents on rice-husk flour reinforced polypropylene composites." Composite Structures 77, no. 1 (2007): 45-55.

[8]. Cantero, Guillermo, AitorArbelaiz, Rodrigo Llano-Ponte, and Iñaki Mondragon. "Effects of fibre treatment on wettability and mechanical behaviour of flax/polypropylene composites." Composites science and technology 63, no. 9 (2003): 1247-1254.

[9]. Neeli, Naresh, M. P. Jenarthanan, and G. Dileep Kumar. "Multi-response optimization for machining GFRP composites using GRA and DFA." Multidiscipline Modeling in Materials and Structures (2018).

[10]. Mohanty, Smita, Sushil K. Verma, Sanjay K. Nayak, and Sudhansu S. Tripathy. "Influence of fiber treatment on the performance of sisal-polypropylene composites." Journal of Applied Polymer Science 94, no. 3 (2004): 13361345.

[11]. Tajvidi, Mehdi, and GhanbarEbrahimi. "Water uptake and mechanical characteristics of natural fillerpolypropylene composites." Journal of Applied Polymer Science 88, no. 4 (2003): 941-946. 
[12]. Aridi, N. A. M., S. M. Sapuan, E. S. Zainudin, and Faris M. AL-Oqla. "Mechanical and morphological properties of injection-molded rice husk polypropylene composites." International Journal of polymer analysis and characterization 21, no. 4 (2016): 305-313.

[13]. Jenarthanan, M. P., and R. Jeyapaul. "Optimisation of machining parameters on milling of GFRP composites by desirability function analysis using Taguchi method." International journal of Engineering, science and Technology 5, no. 4 (2013): 22-36.

[14]. Jenarthanan, M. P., and R. Jeyapaul. "Machinability study of carbon fibre reinforced polymer (CFRP) composites using design of experiment technique." Pigment \& Resin Technology (2014).

[15]. Sojoudiasli, Helia, Marie-Claude Heuzey, and Pierre J. Carreau. "Mechanical and morphological properties of cellulose nanocrystal-polypropylene composites." Polymer Composites 39, no. 10 (2018): 3605-3617.

[16]. Ayrilmis, Nadir, AlperenKaymakci, and FerhatOzdemir. "Physical, mechanical, and thermal properties of polypropylene composites filled with walnut shell flour." Journal of Industrial and Engineering Chemistry 19, no. 3 (2013): 908-914.

[17]. Ramesh, S., and M. P. Jenarthanan. "Investigating the performance of powder mixed electric discharge machining of Nimonic 75 by using different tool materials." World Journal of Engineering (2018).

[18]. Unterweger, Christoph, Tina Mayrhofer, Francesco Piana, Jiri Duchoslav, David Stifter, Claudia Poitzsch, and Christian Fürst. "Impact of fiber length and fiber content on the mechanical properties and electrical conductivity of short carbon fiber reinforced polypropylene composites." Composites Science and Technology 188 (2020): 107998.

[19]. Panthapulakkal, Suhara, and MohiniSain. "Studies on the water absorption properties of short hemp-glass fiber hybrid polypropylene composites." Journal of Composite Materials 41, no. 15 (2007): 1871-1883.

[20]. Zaman, Haydar U., and M. D. H. Beg. "Preparation, structure, and properties of the coir fiber/polypropylene composites." Journal of Composite Materials 48, no. 26 (2014): 3293-3301.

[21]. NeusAnglès, M., Joan Salvadó, and Alain Dufresne. "Steam-exploded residual softwood-filled polypropylene composites." Journal of Applied Polymer Science 74, no. 8 (1999): 1962-1977.

[22]. Liang, J. Z., and F. H. Li. "Measurement of thermal conductivity of hollow glass-bead-filled polypropylene composites." Polymer Testing 25, no. 4 (2006): 527-531.

[23]. Munde, Yashwant S., Ravindra B. Ingle, and I. Siva. "Effect of sisal fiber loading on mechanical, morphological and thermal properties of extruded polypropylene composites." Materials Research Express 6, no. 8 (2019): 085307.

[24]. Colucci, Giovanna, Hadrien Simon, DavideRoncato, BrunettoMartorana, and Claudio Badini. "Effect of recycling on polypropylene composites reinforced with glass fibres." Journal of Thermoplastic Composite Materials 30, no. 5 (2017): 707-723.

[25]. Jenarthanan, M. P., R. Gokulakrishnan, B. Jagannaath, and P. Ganesh Raj. "Multi-objective optimization in end milling of GFRP composites using Taguchi techniques with principal component analysis." Multidiscipline Modeling in Materials and Structures (2017).

[26]. Pallavi, D. R., and Anasuya Rai. "Bancassurance Prototypes in Indian Perspective: A Contemporary Evaluation." Review of Management 8, no. 3/4 (2018): 35-39.

[27]. Sai Sachidhananda, S., Khyati Shetty, and D. R. Pallavi. "Mercenary-A Mirage of Success: A Case Study with Reference to International Arena." International Journal of Applied Business and Economic Research 15 (2017): 119-126.

[28]. Lokhande, Amol, C. Venkateswaran, M. Ramachandran, C. Sathiyaraj, and K. Nathiya. "Recycling Process Impact in Current Scenario Manufacturing: A Study." indicators 1 (2011): 0-6.

[29]. Jain, Mayank, and M. Ramachandran. "Buy/Make decision making framework for pump product development with multi criteria decision making." International Journal of Applied Engineering Research 10, no. 11 (2015): 10486-9.

[30]. Fegade, vishal, u. Ragavendran, and m. Ramachandran. "numerical investigation of hybrid helical spring for total deformation and von mises analysis."

[31]. Nemade, Varsha, Bhavna Sharma, M. Ramachandran, and Vishal Fegade. "Developing communication skill in a rural pharmacy college." Journal of Pharmacy Research 12, no. 4 (2018): 536.

[32]. Rawal, Shannay, M. Ramachandran, Vishal Fegade, and U. Ragavendran. "Simplification of Home Automation System Using Various Sensors, Actuators and Control System." International Journal of Engineering \& Technology 7, no. 3.1 (2018): 174-177.

[33]. Rezaei, Fateme, R. Yunus, and N. A. Ibrahim. "Effect of fiber length on thermomechanical properties of short carbon fiber reinforced polypropylene composites." Materials \& Design 30, no. 2 (2009): 260-263.

[34]. Jayaraman, Krishnan. "Manufacturing sisal-polypropylene composites with minimum fibre degradation." Composites Science and technology 63, no. 3-4 (2003): 367-374.

[35]. Kittur, Jeevan, M. RAMACHANDRAN, Vishal Fegade, and U. Ragavendran. "Numerical Investigation of Total Deformation Inroller Bearing using Ansys Analysis." International Journal of Mechanical and Production Engineering Research and Development (IJMPERD): 51-58.

[36]. Ramachandran, M., U. Ragavendran, and Vishal Fegade. "Selection of Used Piston for Remanufacturing Using Fuzzy TOPSIS Optimization." In Fuzzy Systems and Data Mining IV, pp. 61-67. IOS Press, 2018.

[37]. Fegade, Vishal, Shannay Rawal, and M. Ramachandran. "Metamodel-based parametric study of composite laminates." In IOP Conference Series: Materials Science and Engineering, vol. 810, no. 1, p. 012051. IOP Publishing, 2020. 
[38]. Ramachandran, M., Vishal Fegade, and U. Ragavendran. "Parameters Optimisation For Drilling Of Austenitic Stainless Steel By Taguchi Method Using Desirability Function Analysis." Technology 8, no. 11 (2017): $229-237$.

[39]. Ragavendran, U., Viral Mehta, Vishal Fegade, and M. Ramachandran. "Dynamic Analysis of Single Fold Symmetric Composite Laminates." international Journal of civil Engineering and Technology 8, no. 11 (2017): 536545.

[40]. Ramachandran, M., Vishal Fegade, and P. P. Raichurkar. "Strategy Performance Evaluation of a Port Organisation based on Multi-Criteria Decision Making using Fuzzy Logic Method." NMIMS Management Review 33 (2017): 27 34.

[41]. Sakthivel, M., S. Vijayakumar, and M. P. Jenarthanan. "Grey-fuzzy logic to optimise process parameters in drilling of glass fibre reinforced stainless steel mesh polymer composite." Pigment \& Resin Technology (2017).

[42]. Qian, Yong, Ping Wei, Pingkai Jiang, JianweiHao, and Jianxin Du. "Preparation of hybrid phosphamide containing polysilsesquioxane and its effect on flame retardancy and mechanical properties of polypropylene composites." Composites Part B: Engineering 45, no. 1 (2013): 1541-1547.

[43]. Cesano, Federico, Mohammed Jasim Uddin, Alessandro Damin, and DomenicaScarano. "Multifunctional conductive paths obtained by laser processing of non-conductive carbon nanotube/polypropylene composites." Nanomaterials 11, no. 3 (2021): 604. 